\title{
Development of hepatopulmonary syndrome during combination therapy for portopulmonary hypertension
}

\author{
To the Editor:
}

Portopulmonary hypertension (PoPH) and hepatopulmonary syndrome (HPS) are rare pulmonary vascular complications of portal hypertension [1]. In PoPH, pulmonary vascular resistance (PVR) is elevated, resulting in heightened right ventricular workload which may lead to right-sided heart failure. HPS is characterised by progressive hypoxaemia due to diffusion-perfusion mismatching caused by regional, mostly basilar, intrapulmonary vascular dilatations of capillary and pre-capillary vessels. The exact pathogenesis of both conditions is unclear, as is the underlying predisposition causing a small proportion of patients with liver disease to develop PoPH or HPS.

Although few cases of simultaneous occurrence of PoPH and HPS have been reported [2,3], most patients present with distinct syndromes, i.e. either PoPH or HPS. Of note, there are several case reports demonstrating resolution of HPS with the development of PoPH [4-6], suggesting that the development of an obliterating pulmonary vasculopathy may result in redistribution of pulmonary blood flow away from areas where intrapulmonary vascular dilatations are abundant. To the best of our knowledge, there are no reports on the development of HPS in patients undergoing medical treatment of PoPH. We here report on the clinical course of a patient initially presenting with severe PoPH who had resolution of pulmonary hypertension with combined pulmonary vasodilator therapy, but developed HPS at the same time.

This 56-year-old woman with a history of cirrhosis (Child-Pugh class B) and portal hypertension due to autoimmune hepatitis was admitted to our hospital because of progressive dyspnoea on exertion and right-sided heart failure with extensive fluid retention. On admission, she presented in World Health Organization functional class (FC) IV and was unable to walk. Echocardiography showed severely impaired right heart function. She was mildly hypoxaemic but contrast-enhanced echocardiography with agitated saline showed no appearance of microbubbles in the left heart, thus excluding the presence of HPS at that time. Right heart catheterisation revealed severe pulmonary hypertension (table 1). Other causes of pre-capillary pulmonary hypertension were ruled out and a diagnosis of $\mathrm{PoPH}$ was made. Combination therapy with the phosphodiesterase-5 inhibitor tadalafil and the endothelin-receptor antagonist macitentan was initiated.

Over the following weeks, the patient's clinical condition improved substantially (table 1). After 2 months, she presented in FC II with a 6-min walk distance of more than $400 \mathrm{~m}$. After 6 months, N-terminal proB-type natriuretic peptide (NT-proBNP) dropped to near normal levels and echocardiography showed ameliorated right ventricle function. After 1 year, however, exertional dyspnoea started to increase again. At this time, hypoxaemia worsened, and the patient developed orthodeoxia with an adequate increase in arterial oxygen tension while breathing $100 \%$ oxygen (table 1). Pulmonary function testing showed normal lung volumes and the diffusion capacity for carbon monoxide was $73 \%$ of the predicted value. Computed tomography of the chest showed no signs of parenchymal lung disease or pleural effusions, but revealed dilated pulmonary vessels in the basilar lung zones. NT-proBNP remained low and echocardiography showed normalised right heart function. On right heart catheterisation, pulmonary artery pressures were near normal with a high cardiac output and a normal PVR (table 1). Now, contrast-enhanced

@ERSpublications

Patients with portopulmonary hypertension may develop hepatopulmonary syndrome during treatment with pulmonary vasodilators http://ow.ly/brHB30mOIWn

Cite this article as: Olsson KM, Meyer K, Berliner D, et al. Development of hepatopulmonary syndrome during combination therapy for portopulmonary hypertension. Eur Respir J 2019; 53: 1801880 [https://doi. org/10.1183/13993003.01880-2018]. 


\section{TABLE 1 Patient characteristics}

\begin{tabular}{|c|c|c|c|c|c|}
\hline & $\begin{array}{l}\text { Baseline } \\
06 / 2017\end{array}$ & $\begin{array}{c}\text { Follow up } \\
09 / 2017\end{array}$ & $\begin{array}{l}\text { Follow up } \\
01 / 2018\end{array}$ & $\begin{array}{l}\text { Follow up } \\
07 / 2018\end{array}$ & $\begin{array}{c}\text { Follow up } \\
08 / 2018\end{array}$ \\
\hline Functional class & IV & ॥ & II & III & III \\
\hline PAH therapy & & Tadalafil/macitentan & Tadalafil/macitentan & Tadalafil/macitentan & Tadalafil \\
\hline NT-proBNP $n g \cdot L^{-1}$ & 7086 & & 198 & 171 & 131 \\
\hline Right atrial pressure $\mathrm{mmHg}$ & 15 & & & 7 & \\
\hline Cardiac output L- $\min ^{-1}$ & 4.3 & & & 9.4 & \\
\hline Cardiac index $\mathrm{L} \cdot \mathrm{min}^{-1} \cdot \mathrm{m}^{-2}$ & 2.3 & & & 5.3 & \\
\hline PVR dyn $\cdot s \cdot \mathrm{cm}^{-5}$ & 706 & & & 102 & \\
\hline Mixed venous oxygen saturation $\%$ & 55 & & & 77 & \\
\hline $\mathrm{PaO}_{2} \mathrm{mmHg}$ (upright/supine/ $100 \%$ oxygen) & $74 /-/-$ & $64 /-/-$ & $57 /-/-$ & $55 / 62 / 546$ & $60 /-/-$ \\
\hline $\mathrm{PaCO}_{2} \mathrm{mmHg}$ & 29 & 31 & 31 & 29 & 33 \\
\hline
\end{tabular}

PAH: pulmonary arterial hypertension; NT-proBNP: N-terminal pro-B-type natriuretic peptide; PVR: pulmonary vascular resistance; $P_{a O_{2}}$ : arterial oxygen tension; $\mathrm{PaCO}_{2}$ : arterial carbon dioxide tension.

echocardiography disclosed severe intrapulmonary shunting (>100 microbubbles appearing in the left atrium and the left ventricle after four cardiac cycles), confirming the diagnosis of HPS [7, 8].

We interpreted these findings as complete resolution of PoPH complicated by development of HPS, both associated with the combined use of pulmonary vasodilator therapy. Therefore, macitentan was stopped and tadalafil was continued as monotherapy. 6 weeks later, the patient was still in FC III with slight improvement of her blood gases, while NT-proBNP remained low and echocardiography continued to show normal right ventricle function. At present, the patient is being evaluated for liver transplantation.

In summary, our case illustrates that HPS can develop as a consequence of the use of pulmonary vasodilators in patients with PoPH. We cannot exclude the possibility that drug-mediated pulmonary vasodilation was the direct cause of HPS, although we believe that it is more likely that pre-existing HPS was masked by the presence of severe pulmonary hypertension and unmasked by its successful treatment. It is important that physicians are aware that HPS may develop during medical treatment of $\mathrm{PoPH}$ and monitor their patients accordingly. In addition, it is possible that the risk of developing HPS is particularly high in patients with $\mathrm{PoPH}$ who receive combination therapy with pulmonary vasodilators, but this hypothesis needs to be addressed in future clinical trials.

Karen M. Olsson ${ }^{1}$, Katrin Meyer $^{1}$, Dominik Berliner ${ }^{2}$ and Marius M. Hoeper $\odot^{1}$

${ }^{1}$ Dept of Respiratory Medicine and German Centre for Lung Research (DZL/BREATH), Hannover Medical School, Hannover, Germany. ${ }^{2}$ Dept of Cardiology and Angiology, Hannover Medical School, Hannover, Germany.

Correspondence: Karen M. Olsson, Dept of Respiratory Medicine, Hannover Medical School, Carl-Neuberg-Str. 1, 30625 Hannover, Germany. E-mail: olsson.karen@mh-hannover.de

Received: Oct 032018 | Accepted after revision: Nov 142018

Conflict of interest: K.M. Olsson reports personal fees for speaking/consultations from Actelion. K. Meyer has nothing to disclose. D. Berliner has nothing to disclose. M.M. Hoeper reports personal fees for speaking/consultations from Actelion, Bayer, GSK and Merck.

\section{References}

1 Hoeper MM, Krowka MJ, Strassburg CP. Portopulmonary hypertension and hepatopulmonary syndrome. Lancet 2004; 363: 1461-1468

2 Pham DM, Subramanian R, Parekh S. Coexisting hepatopulmonary syndrome and portopulmonary hypertension: implications for liver transplantation. J Clin Gastroenterol 2010; 44: e136-e140.

3 Tasaka S, Kanazawa M, Nakamura H, et al. [An autopsied case of primary pulmonary hypertension complicated by hepatopulmonary syndrome]. Nihon Kyobu Shikkan Gakkai Zasshi 1995; 33: 90-94.

4 Umeda A, Tagawa M, Kohsaka T, et al. Hepatopulmonary syndrome can show spontaneous resolution: Possible mechanism of portopulmonary hypertension overlap? Respirology 2006; 11: 120-123.

5 Zopey R, Susanto I, Barjaktarevic I, et al. Transition from hepatopulmonary syndrome to portopulmonary hypertension: a case series of 3 patients. Case Rep Pulmonol 2013; 2013: 561870. 
6 Mal H, Burgiere O, Durand F, et al. Pulmonary hypertension following hepatopulmonary syndrome in a patient with cirrhosis. J Hepatol 1999; 31: 360-364.

7 Krowka MJ, Fallon MB, Kawut SM, et al. International Liver Transplant Society practice guidelines: diagnosis and management of hepatopulmonary syndrome and portopulmonary hypertension. Transplantation 2016; 100: $1440-1452$.

8 Tonelli AR, Naal T, Dakkak W, et al. Assessing the kinetics of microbubble appearance in cirrhotic patients using transthoracic saline contrast-enhanced echocardiography. Echocardiography 2017; 34: 1439-1446.

Copyright @ERS 2019 